\title{
Apoptosis mediates decrease in cellularity during the regression of Arthus reaction in cornea
}

\author{
Noriko Ozaki, Masamichi Ishizaki, Mohammad Ghazizadeh, Nobuaki Yamanaka
}

\begin{abstract}
Backgroundlaims-The Arthus type allergic reaction is characterised by inflammatory cell infiltration and marked neovascularisation in the cornea. During the healing stages, inflammatory cells and newly formed microvessels gradually disappear. The aim was to establish whether apoptosis affected the regression of inflammatory cells and newly formed microvessels, in order to define more clearly the cellular mechanisms involved in the pathobiology of corneal diseases.
\end{abstract}

Methods-Albino male rabbits were injected subcutaneously with $5 \mathrm{mg} / \mathrm{ml}$ bovine serum albumin (BSA) incorporated in Freund's complete adjuvant twice weekly. Under the anaesthesia, $30 \mu 1$ of a $0.5 \mathrm{mg} / \mathrm{ml} \mathrm{BSA}$ solution was injected into the central corneal stroma to induce an Arthus type allergic reaction. The injured corneas were collected at various time points ranging from 3 to 20 days. Apoptotic cells were identified by both light microscopy using in situ TdT-dUTP nick end labelling (TUNEL) method and electron microscopy.

Results-With increasing time after induction of the Arthus reaction, marked neovascularisation and infiltrated inflammatory cells such as polymorphonuclear cells (PMNs) and plasma cells were observed in the cornea. Thereafter, the inflammatory cells and newly formed microvessels gradually disappeared. Coincidently, the numbers of microvessel endothelial cells and infiltrated inflammatory cells undergoing apoptosis were increased. Apoptotic bodies were taken up by macrophages, PMNs, as well as myofibroblasts derived presumably from transformation of migrated keratocytes.

Conclusions-These data demonstrate that regression of the cellular infiltrates and microvessel endothelial cells associated with the Arthus reaction in the cornea occurs via apoptosis. This finding adds insights into the cellular mechanisms regulating the pathobiology of corneal diseases.

(Br f Ophthalmol 2001;85:613-618)

The avascular cornea has highly regular orientation of stromal collagen fibres that enhances optical transparency, and makes it distinct from other tissues in the body. Inflammation in the cornea differs in many respects from that in other body sites. Inflammatory cells may infiltrate the cornea from various sites, such as conjunctival and scleral vessels, via the limbus, and less commonly from the anterior chamber. ${ }^{1}$ When oedema is accompanied by inflammation, a portion of the disruptive light scattering is caused by infiltration of the stroma by inflammatory cells and by exudates derived from the limbal vessels. The degree of opacification and visual disturbance appears to be related to the number and density of inflammatory cells and their location, and to the presence or absence of associated enzymatically induced stromal damage, vascularisation, and calcium deposition. ${ }^{2}$ To maintain corneal transparency, it is necessary to eliminate the infiltrated inflammatory cells. On the other hand, corneal neovascularisation is a sequel of numerous inflammatory lesions of the ocular anterior segment. ${ }^{3}$ In addition, not only corneal neovascularisation but also ocular neovascularisation such as that occurring in diabetic proliferative retinopathy, neovascular glaucoma, and choroidal neovascularisation can cause severe disturbances of ocular function. Therefore, it is highly imperative to elucidate the mechanisms underlying the development, as well as, regression, of neovascularisation.

Recently, one form of cell death referred to as "programmed cell death" mediated by a process termed "apoptosis" has been recognised. Apoptosis plays an important part during tissue development, maintaining homeostasis, response to infection and wound healing by eliminating unwanted cells. ${ }^{4}$ Meeson et al reported that regression of capillary channels in pupillary membrane is caused by apoptosis. ${ }^{6}$ Wilson and Kim suggested that keratocyte apoptosis after corneal epithelial injury is an initiator of the wound healing response. ${ }^{7} \mathrm{~A}$ characteristic feature of apoptosis is recognised as DNA digestion at internucleosomal sites, producing small double stranded fragments of DNA that migrate in a ladder pattern (multiple of $185 \mathrm{bps}$ ) after electrophoresis in agarose gels. Moreover, apoptosis is identified morphologically by electron microscopy, or by light microscopy using the in situ TdT-dUTP nick end labelling (TUNEL) method. Typical morphological changes in apoptosis include cell shrinkage and loss of normal contacts, dense chromatin condensation, cellular budding, and fragmentation. Finally, apoptotic cells are normally eliminated by macrophages or neighbouring cells without an inflammatory response. $^{8}$

The rabbit cornea provides an ideal site for studying the in vivo consequences of antigenantibody reactions and diffusion of the newly formed microvessels as it is transparent and avascular. Germuth et al reported that the
Accepted for publication 16 October 2000 

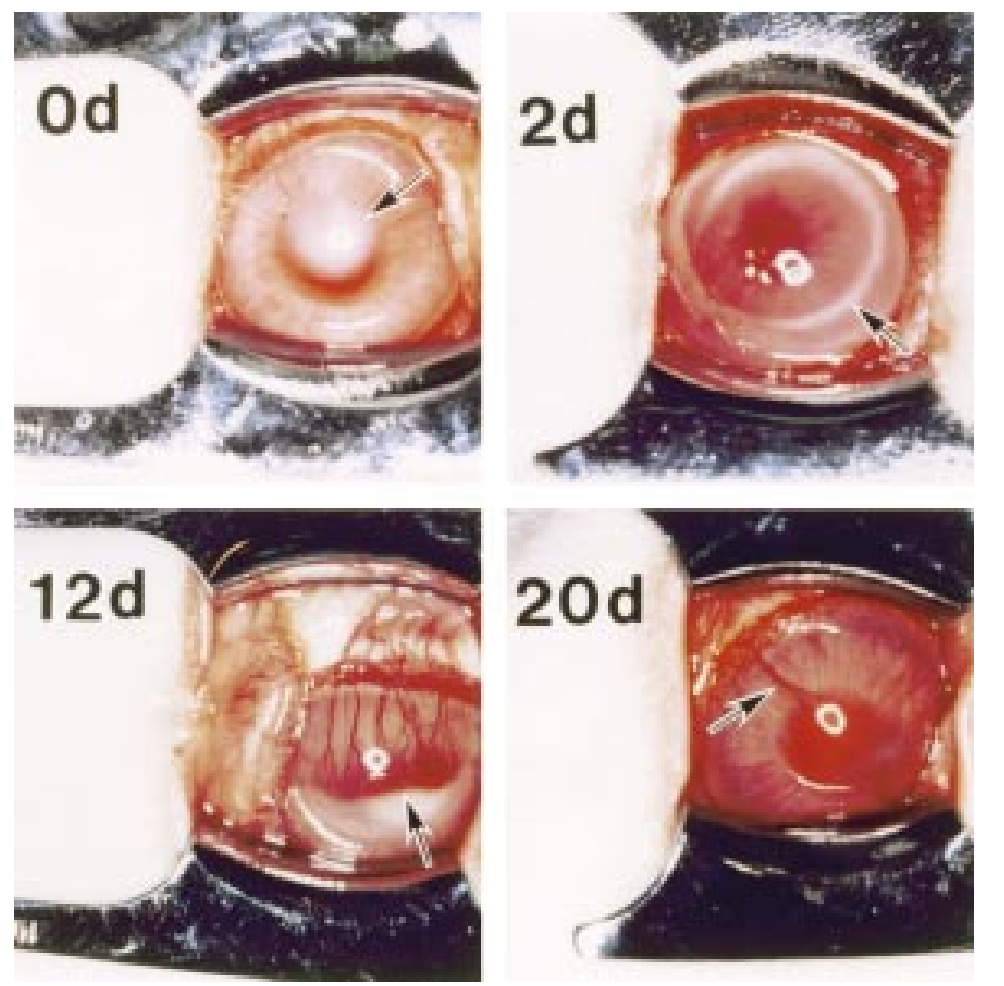

Figure 1 Sensitised rabbit corneas showing opacity as a result of injection (arrow) at day $O$ (Od), immuno-ring (arrow) at 2 days (2d) after injection and induction of neovascular response. Newly formed microvessels (arrow) reached to the centre of the cornea at 12 days (12d). These microvessels (arrow) gradually diminished at the healing stage on day 20 (20d).

injection of BSA into the cornea of homologously sensitised rabbit induced an immunoring (Arthus reaction), and inflammatory cell infiltrates and newly formed microvessels were also observed. Subsequently, the inflammatory cells and neovascularisation gradually disappeared during the healing stages. However, the mechanisms that govern the cellularity and vascularity in Arthus type allergic reaction remain undefined. In the present study, we hypothesised that programmed cell death or apoptosis of endothelial and inflammatory cells might play a major part in resolving the Arthus type reaction. Indeed, recently studies have indicated that apoptosis is associated with the involution of capillary networks..$^{11}$

\section{Methods}

INDUCTION OF ARTHUS REACTION IN CORNEAS Albino male rabbits weighing $2.5-3 \mathrm{~kg}$ were purchased from Saitama Experimental Animal Supply (Saitama, Japan). Animal experiments were performed in compliance with the experimental animal ethics review committee of Nippon Medical School, Japan. As described previously, ${ }^{9}$ animals were injected subcutaneously weekly twice with $5 \mathrm{mg} / \mathrm{ml}$ bovine serum albumin (BSA) incorporated in Freund's complete adjuvant. Thereafter, the rabbits were injected with $2 \mathrm{mg} / \mathrm{ml} \mathrm{BSA}$ as a booster weekly two or three times. Rabbits whose antibody titre for BSA had risen more than 16-fold by the Ouchterlony method were used for this study. To induce the Arthus type allergic phenomenon, $30 \mu \mathrm{l}$ of an $0.5 \mathrm{mg} / \mathrm{ml}$ BSA solution was injected in the central corneal stroma of the unilateral eye under the anaesthesia with sodium thiopentone (Ravonal). Control animals were injected with $30 \mu \mathrm{l}$ sterile normal saline in the central corneal stroma of the unilateral eye. The corneas were observed during the experimental period by direct visual examination. The rabbits were sacrificed with an overdose of sodium thiopentone intravenously at various time points ranging from 3 to 20 days. At each time point, three to four injured corneal buttons with adjacent sclera were collected and processed for light and electron microscopic examinations.

\section{LIGHT MICROSCOPY}

Corneal tissue specimens were fixed in $4 \%$ buffered paraformaldehyde for 16-24 hours at $4^{\circ} \mathrm{C}$. Each tissue was divided into two halves. One semicircular half was embedded in paraffin and the other was immersed in OCT compound and frozen in dry ice-acetone. A section from each paraffin block was stained with haematoxylin and eosin for histological examinations.

MORPHOLOGICAL IDENTIFICATION OF APOPTOSIS Apoptotic cells were assessed morphologically by both light and electron microscopy. For light microscopy, a modification of the TUNEL method $^{12}$ was used to detect the apoptotic cells, and a simultaneous immunostaining for thrombomodulin (TM) was performed to identify the microvessels. ${ }^{13-15}$ Briefly, $4 \mu \mathrm{m}$ thick frozen sections were incubated with $20 \mu \mathrm{g} / \mathrm{ml}$ proteinase $\mathrm{K}$ for 15 minutes at room temperature and washed in distilled water. The sections were rinsed in TdT buffer $(30 \mathrm{mM}$ TRIS-HCl, pH 7.2, $140 \mathrm{mM}$ sodium cacodylate, $1 \mathrm{mM}$ cobalt chloride) and incubated with $0.5 \mathrm{U} / \mu \mathrm{l} \mathrm{TdT}$ (Gibco BRL; Rockville, $\mathrm{MD}, \mathrm{USA}$ ) and $10 \mu \mathrm{M}$ fluorescein-12-dUTP (Boehringer Mannheim, Germany) in TdT buffer for 60 minutes at $37^{\circ} \mathrm{C}$. The sections were washed with PBS four times each for 5 minutes and incubated with goat anti-rabbit TM (provided by Dr David Stern, Columbia University) at 1:800 dilution. After washing three times with PBS, the sections were incubated with swine anti-goat IgG labelled with Texas red (EY Laboratories, San Mateo, CA, USA) at 1:50 dilution, washed three times with PBS, and cover slipped using Vectashield H-1000 mounting medium (Vector Laboratories, Inc, Burlingame, CA, USA).

INTERPRETATION OF STAINING

Examination of the stained sections and photography were performed with a fluorescence microscope (Model BX 60, Olympus, Tokyo, Japan). The number of apoptotic cells was assessed semiquantitatively. TUNEL positive endothelial cells of microvessels and inflammatory cells in each area were counted in five microscopic fields at $400 \times$ magnification and averaged.

ELECTRON MICROSCOPY

For the electron microscopic study, the excised corneas were cut into small pieces and fixed in $2.5 \%$ glutaraldehyde in $0.1 \mathrm{M}$ phosphate 


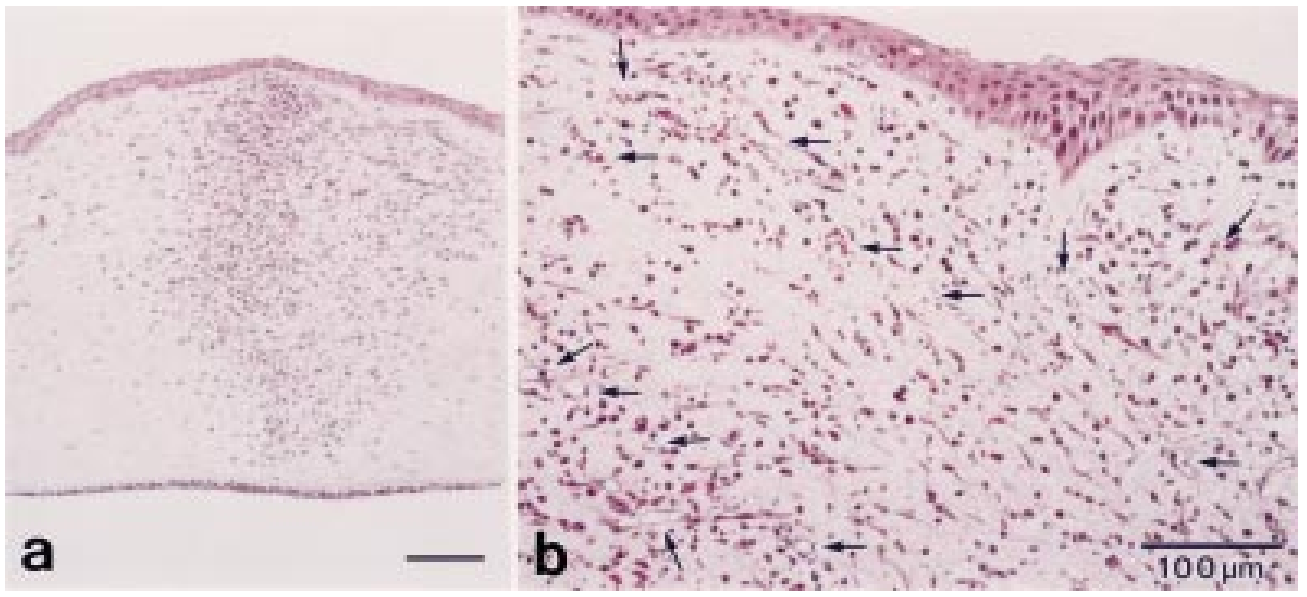

Figure 2 Light microscopic observation (haematoxylin eosin staining) at 3 days. (a) The infiltration of inflammatory cells mainly polymorphonuclear neutrophils (PMNs) was noted. (b) At the limbus newly formed dense capillaries were observed (arrows).

buffer, $\mathrm{pH} 7.4$, overnight at $4^{\circ} \mathrm{C}$. After washing in $0.1 \mathrm{M}$ phosphate buffer, the tissue blocks were postfixed with $1 \% \mathrm{OsO}_{4}$ in $0.1 \mathrm{M}$ phosphate buffer, $\mathrm{pH} 7.4$, for 2 hours, dehydrated, and embedded in Epok 821.

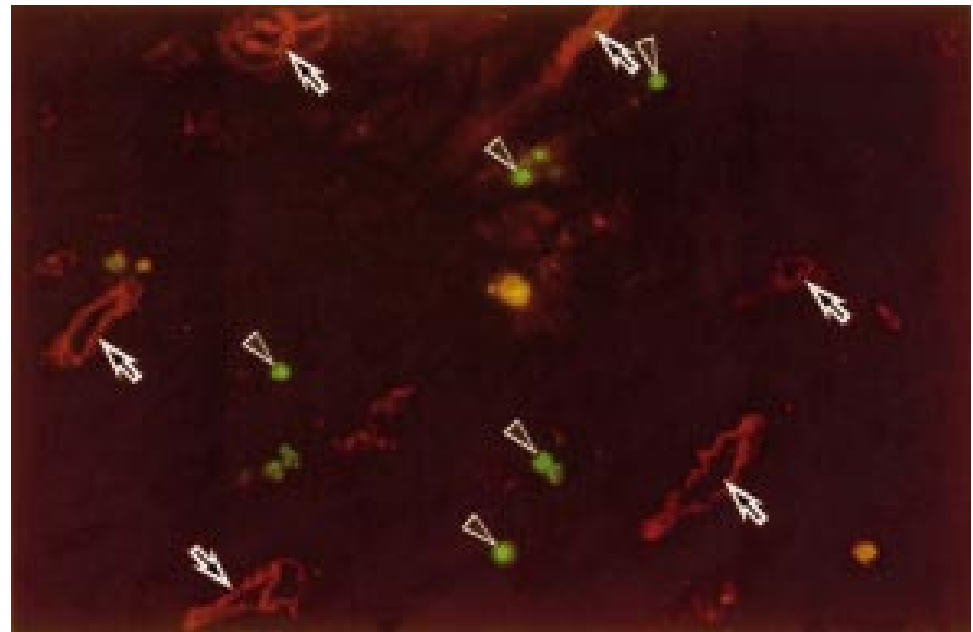

Figure 3 Double staining for apoptotic cells, and microvessels in the same section at 3 days after injection of BSA. TUNEL positive cells (green, arrowheads) were present in the stroma. However, there were no positive cells in the microvessels as depicted by positive reaction for $T M$ (red, arrows).

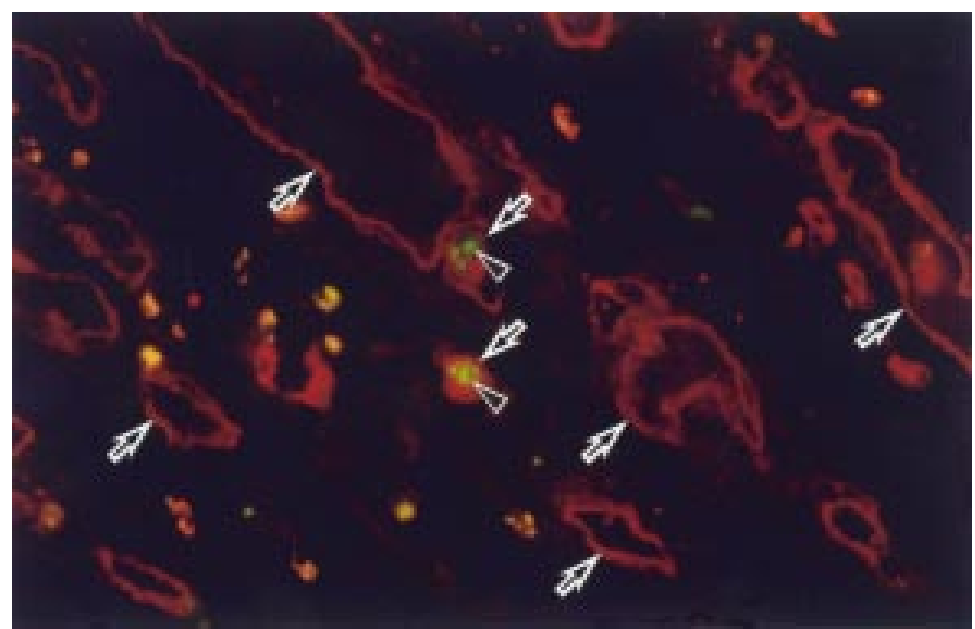

Figure 4 Double staining for apoptotic cells, and microvessels in the same section at 12 days after injection of BSA. Co-localisation of TM expression (red, arrows) and TUNEL positivity (green, arrowheads) is seen in the endothelium.
Ultrathin sections were stained with uranyl acetate and lead citrate, then examined with a Hitachi H-7100 electron microscope at an accelerating voltage of $75 \mathrm{kV}$.

\section{Results}

GROSS EXAMINATION

Immediately after injection of BSA solution, the cloudiness of the cornea as a result of the injection was observed (Fig 1, 0d). Several hours after injection, the cloudiness was resolved but not cleared up completely. At 2 days after injection, an immuno-ring was induced by precipitation of immune complexes (Fig 1, 2d). At day 3, the neovascular response originating from the limbal vessels was evident. At day 9, extensive newly formed microvessels extended towards the immuno-ring, and at the end of second week reached the centre of the cornea with an increase in the central portion and a decrease around the limbus (Fig 1, 12d). In the areas through which the network of newly formed microvessels had transited, the cloudiness disappeared. By the end of 3 weeks, these microvessels were also gradually diminished (Fig 1, 20d).

\section{LIGHT MICROSCOPY}

At 2 days, the corneas that formed immunoring were oedematous and demonstrated the migration of inflammatory cells. By the fluorescent antibody method, rabbit IgG and C3 deposits were present coincidently with the immuno-ring (data not shown). By TUNEL assay, no cell stained positively. At day 3, infiltration of inflammatory cells was noted in association with the immuno-ring (Fig 2a), and at the limbus extensive newly formed microvessels were observed (Fig 2b). TUNEL positive cells were present in the stroma (Fig 3, arrowheads), but there were no such cells in the microvessels (Fig 3, arrows). The area of hypercellularity including capillaries and inflammatory cells gradually translocated from the periphery to the centre of the cornea. After several days, TUNEL positive cells in the microvessel wall began to appear (Fig 4, arrowheads). They were observed frequently in the area circumjacent to the limbus side of the 
capillary accumulation, and less at the periphery of this area. TUNEL positive cells could still be observed in the stroma at the end stage

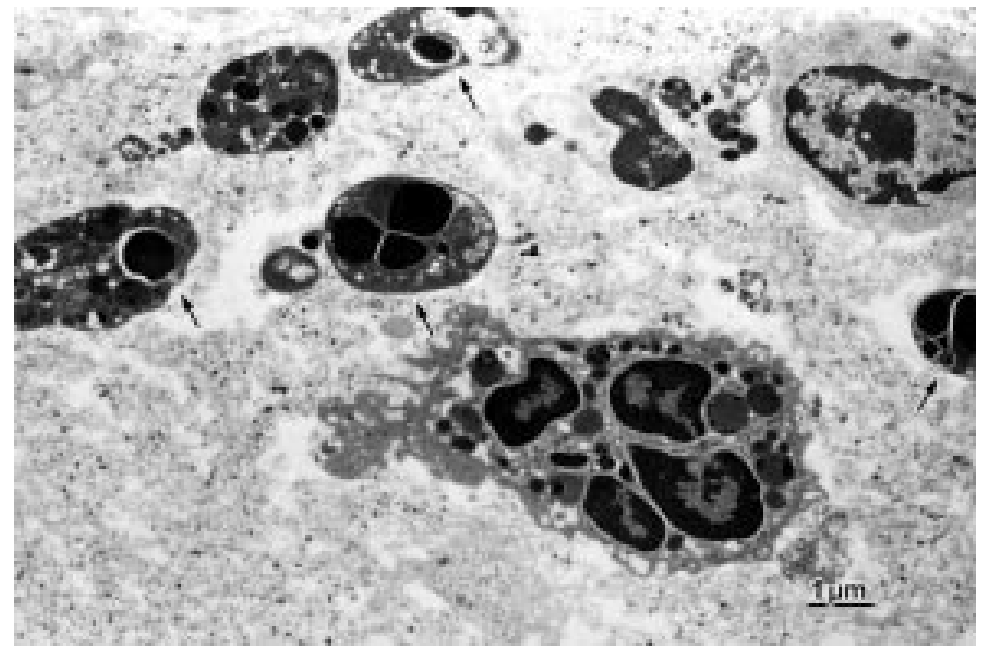

Figure 5 Various types of apoptotic cells and bodies in the stroma are shown (arrows).

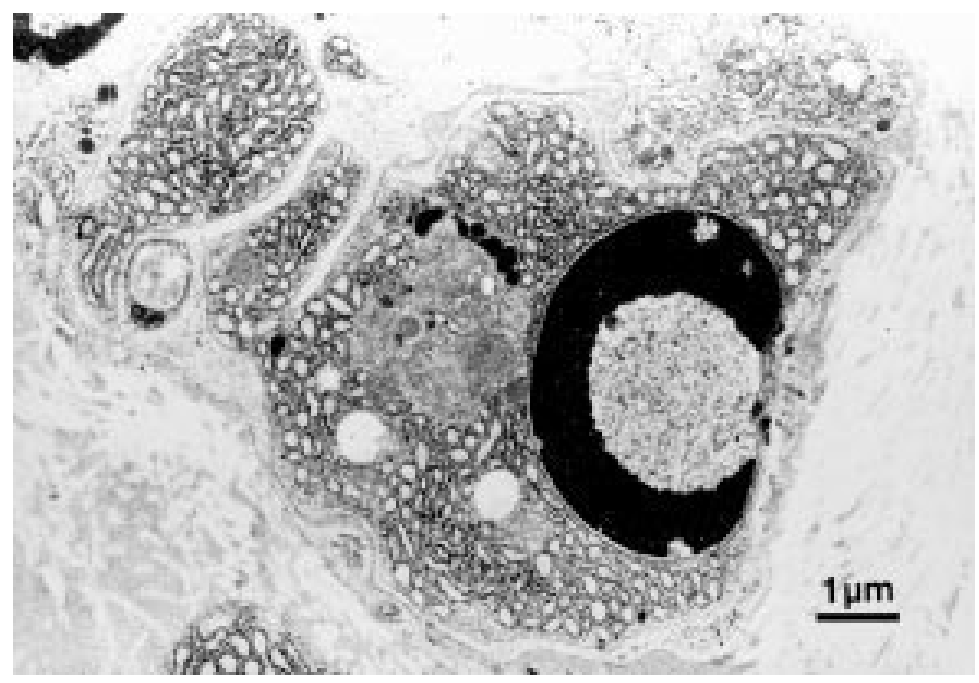

Figure 6 A plasma cell in the early stage of apoptosis at 9 days after injection of BSA.

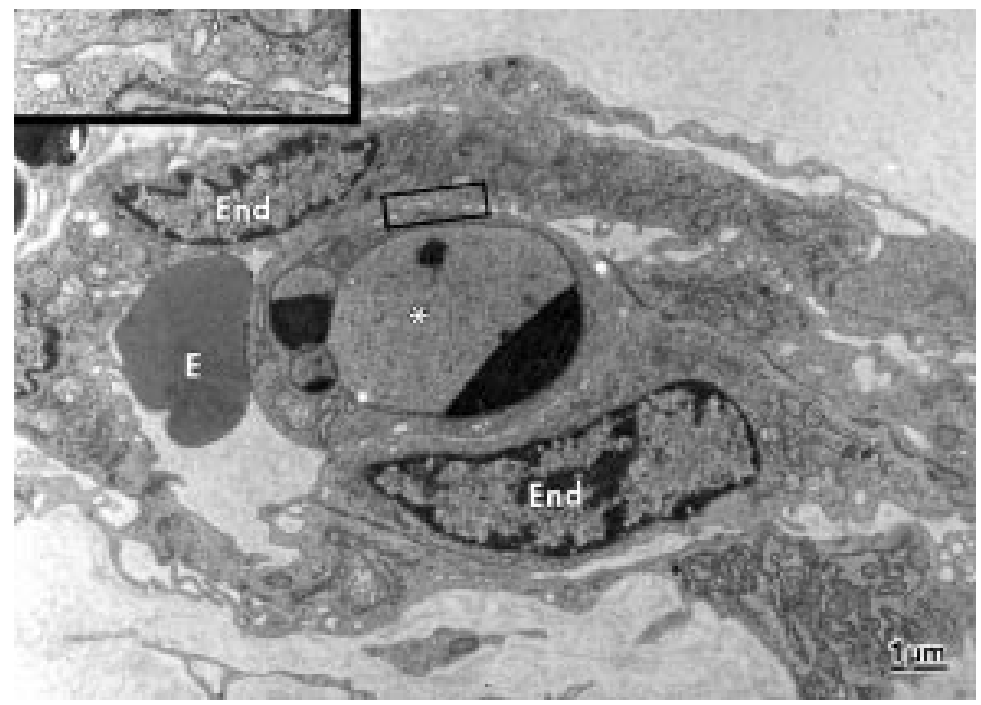

Figure 7 Apoptotic cell in microvessel at 12 days after injection of BSA showing an endothelial cell in the early stage of apoptosis. Note condensation of heterochromatin to form "crescent" in the lobes of the nucleus $\left({ }^{\star}\right)$. Inset shows pinocytic vesicle of endothelial cells (arrows). of inflammation. The accumulation of plasma cells surrounding the microvessels was remarkable, but then decreased in the area through which the network of capillaries had transited.

\section{ELECTRON MICROSCOPY}

By electron microscopy, various types of apoptotic cells and bodies were detected. From 3 days after the challenge, apoptotic neutrophils and bodies containing fragments of chromatin condensation were observed. Excessive apoptotic bodies beyond the capacity of take up by phagocytes were occasionally observed (Fig 5). From day 9, plasma cells showed apoptotic changes (Fig 6). From day 12, newly formed microvessels containing endothelial cells with initial apoptotic changes such as crescent condensation of the chromatin were observed (Fig 7). Apoptotic bodies were found in phagocytic cells (Fig 8). Apoptotic PMNs were phagocytised by macrophages (Fig 8A) and apoptotic neutrophils by PMNs (Fig 8B). Apoptotic bodies were also taken up by fibroblastic cells (Fig 8C).

\section{Discussion}

The cornea is an immune privileged site. ${ }^{16}$ Immune privilege is a term applied to several organs including the brain, testis, and ovary and these sites prohibit the spread of inflammation, since even minor episodes can threaten organ integrity and function.

Recently Griffith et al showed that the induction of apoptosis is a potent mechanism of immune privilege. ${ }^{17}$ In the present study, we observed the developmental process and regression of infiltrated inflammatory cells and newly formed microvessels in the rabbit cornea sensitised with BSA and focused on changes in the corneal cellularity and the relevance of apoptosis in this respect.

At 2 days after the challenge, immuno complex was formed by antigen-antibody reaction, which was observed as an immuno-ring with the naked eyes. Subsequently, the complement system could be activated by the antigenantibody reaction or by the resulting complexes. Such an activation could release complement $\mathrm{C} 3 \mathrm{a}$ and $\mathrm{C} 5 \mathrm{~b}$ fragments and they could induce infiltration of PMNs from the limbal vessels. Previous studies have shown that the membrane attack complex (MAC) which is composed of complement C $5 \mathrm{~b} \sim 9$ fragments promoted the secretion of IL- 8 which induces infiltration of PMNs from human umbilical vein endothelial cells. ${ }^{18}$ This process is thought as a mechanism of PMNs infiltration in the cornea.

The half life of circulating PMNs is estimated to be 5 hours, but that of infiltrated PMNs in tissue is unclear. In either case the ageing PMNs eventually undergo apoptosis. ${ }^{19}$ Furthermore, it is well known that in acute inflammation G-CSF, GM-CSF, interferon $\gamma$, and TNF- $\alpha$ suppressors delay apoptosis of PMNs. ${ }^{20}{ }^{21}$ In this study, the evidence for PMNs undergoing apoptosis was documented at 3 days, but not earlier, such as at 1 day. The same mechanism may be responsible for delayed apoptosis of neutrophils in the present study. 

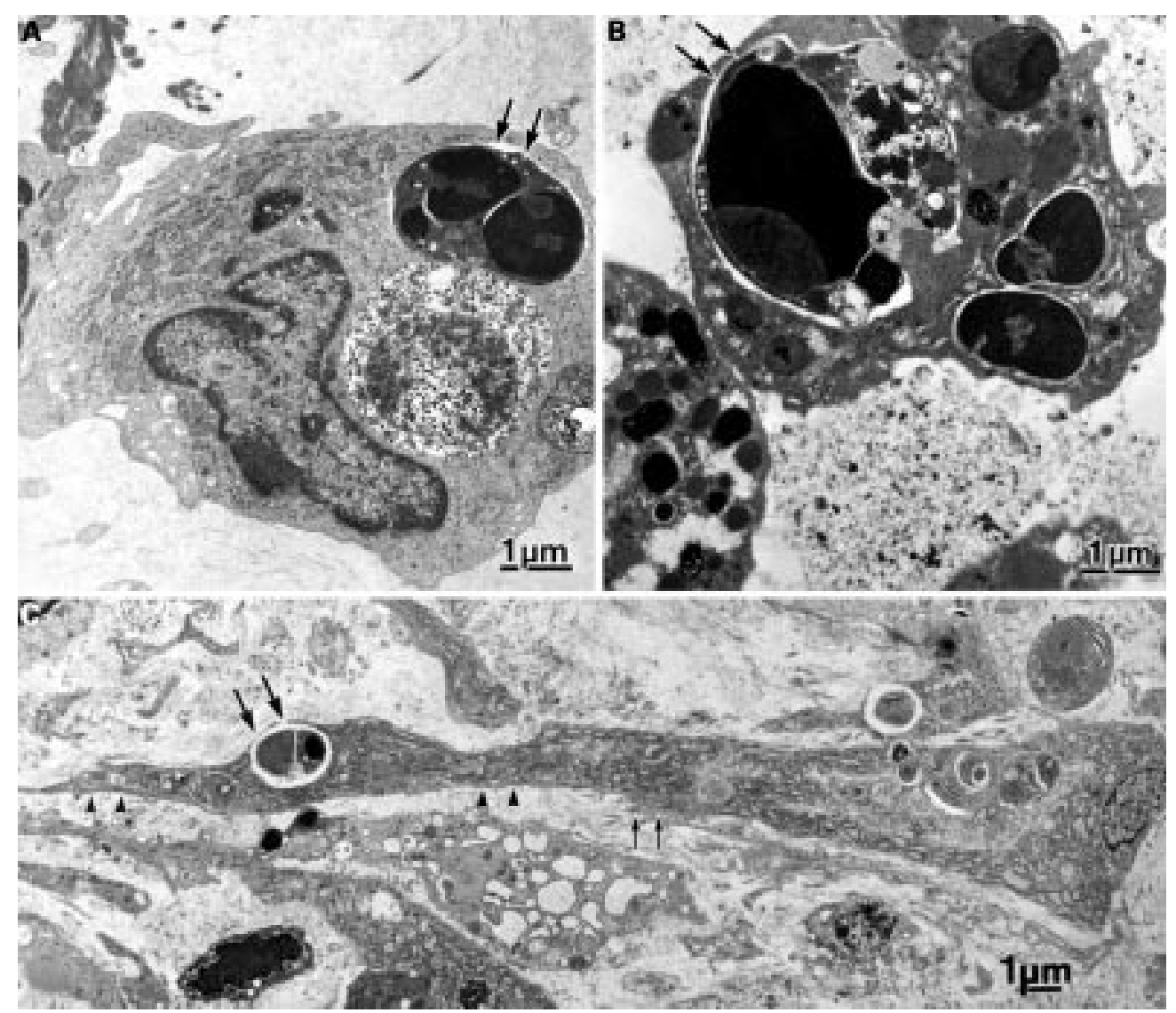

Figure 8 Phagocytosis of apoptotic cells at 12 days after injection of BSA. (A) A macrophage containing apparently a PMN with granules and fragments of chromatin condensation typical of apoptosis (arrows). (B) A PMN containing apparently a neutrophil with granules and fragments of chromatin condensation typical of apoptosis (arrows). (C) Apoptotic body (large arrows) was taken up by fibroblastic cells. This fibroblastic cell has extracellular microtendon associated with the fibronexis (small arrows), electron dense stress fibre (arrowheads), and abundant rough ER, suggesting a myofibroblast.

We observed plasma cells infiltrating around the newly formed microvessels. In general, plasma cells represent the final differentiation stage of B lymphocytes after encountering antigenic agents and interacting with various cell types. The number of plasma cells has to be tightly controlled to avoid overproduction of antibodies. Accordingly, it seems reasonable to speculate that unnecessary plasma cells undergo apoptosis to maintain the balance. In this study, we confirmed in vivo that plasma cells in the cornea underwent apoptosis, particularly in the areas through which the newly formed capillary network had transited. This finding further suggests that apoptosis may participate in the control of antibody producing cells.

From 3 days after the challenge, corneal neovascularisation was observed resulting in more increase in corneal cellularity and consequently the loss of corneal transparency. If corneal neovascularisation persists for a long period of time, it may result in lipid leakage and haemorrhage. After the inflammation subsided, the newly formed microvessels and corneal hypercellularity gradually regressed. We previously confirmed that the endothelial cells of newly formed microvessels in the injured cornea expressed TIMP-2, which is an inhibitor of MMPs (unpublished data). It was also reported that TIMP-2 inhibits neovascularisation. $^{22}$ It is possible that the expression of TIMP-2 may contribute to the regression of newly formed microvessels. Pollman et $a l^{11}$ demonstrated that endothelial cell apoptosis is a prominent feature of in vitro capillary network regression and formation.

In this study, electron microscopy revealed that the endothelial cells of newly formed microvessels underwent apoptosis. This finding suggests that apoptosis reduces the hypercellularity due to the infiltration of PMNs, appearance of plasma cells, and neovascularisation in the cornea, leading to the recovery of the corneal transparency.

At present, it is not clear whether total regression of inflammatory cells in our study is through apoptosis. However, studies on apoptosis of normal cells in the developing rat kidney have shown that uptake of apoptotic cells by phagocytes and degradation of ingested apoptotic cells beyond histological recognition occurs very rapidly (within an hour or so) as a means of cell clearance. ${ }^{23}$ Therefore, low frequencies of histologically demonstrable apoptotic cells reflects clearance of large numbers of cells, particularly since a macrophage can ingest many apoptotic cells. This factor may account for the low frequency of apoptotic cells observed in the histological sections in this study. 
It is usually stated that the apoptotic bodies are often taken up by neighbouring cells, and macrophages do not induce an inflammatory response. ${ }^{8}$ Tsuji et $a l^{24}$ reported that apoptotic human umbilical vein endothelial cells can activate the alternative pathway of the homologous complement and that the complement is related to the clearance of apoptotic cells by phagocytes. On the other hand, myofibroblasts that are characterised by the expression of $\alpha$-smooth muscle actin contribute to the healing of wounds. Recently, it has been reported that during corneal wound healing, the keratocytes that migrate to injured stroma transform into myofibroblasts. ${ }^{25}$ In our study, it was confirmed by electron microscopy that the apoptotic bodies were taken up by macrophages, PMNs, and myofibroblasts. In this process, it is possible that an alternative pathway may be involved, and apoptosis may contribute to the recovery of the corneal transparency. On the other hand, we were unable to detect DNA degradation by electrophoresis, probably due to the overall gradual occurrence of apoptosis. Meanwhile, in the occasionally observed areas with several apoptotic bodies, excessive apoptotic bodies beyond the capacity of take up by phagocytes may undergo secondary necrosis and participate in the prolonged inflammatory sequel there.

Several factors have been proposed as regulators of apoptosis. Fas ligand and tumour necrosis factor (TNF), bind to their receptors and induce apoptosis, while overexpression of bcl-2 blocks it. ${ }^{26}$ Griffith et $a l^{17}$ showed that in the mouse cornea, Fas ligand is expressed on the epithelium and endothelium, suggesting its importance in controlling inflammatory cells entering from the conjunctiva or anterior chamber. Stuart et $a l^{27}$ reported that Fas ligand expressed on human cornea is a critical factor determining the success of orthotopic corneal allografts by inducing cell death in inflammatory cells. It has been reported that all plasma cell lines expressed Fas at a low to moderate level. ${ }^{28}$ As also in the present study, it is possible that by Fas-Fas ligand interaction, inflammatory cells and endothelial cells of newly formed microvessels may undergo apoptosis.

In conclusion, our data demonstrate that regression of the cellular infiltrates and microvessel endothelial cells associated with the Arthus reaction in the cornea occurs via apoptosis. This finding adds insight into the cellular mechanisms regulating the pathobiology of corneal diseases.

This study was supported in part by grant No 08672040 from the Ministry of Education, Science and Culture of Japan, and by the Science Research Promotion Fund from the Promotion and Mutual Aid Corporation for the Private School of Japan. The authors wish to thank Ms Kyoko Wakamatsu for her expert technical assistance.
1 Hinzpeter EN, Naumann GOH. Cornea and sclera. In: Naumann GOH, Apple DJ, eds. Pathology of the eye. New Naumann GOH, Apple DJ, eds. Pa
York; Springer Verlag, 1986:317-41.

2 Spencer WH. Cornea. In: Spencer WH, ed. Ophthalmic pathology. Philadelphia: WB Saunders, 1996:157-333.

3 Kenyon KR, Chaves HV. Morphology and pathologic response of corneal and conjunctival disease. In: Smolin G, Thoft RA, eds. The cornea. Boston: Little, Brown, 1994:69111.

4 Thompson CB. Apoptosis in the pathogenesis and treatment of disease. Science 1995;267:1456-62.

5 Desmouliere A, Badid C, Bochaton-Piallat ML, et al. Apoptosis during wound healing, fibrocontractive diseases and tosis during wound healing, fibrocontractive diseases

6 Meeson A, Palmer M, Calfon M, et al. A relationship between apoptosis and flow during programmed capillary regression is revealed by vital analysis. Development 1996;122:3929-38.

7 Wilson SE, Kim W-J. Keratocyte apoptosis: implications on corneal wound healing, tissue organization, and disease. Invest Ophthalmol Vis Sci 1998;39:220-6.

8 Majno G, Joris I. Apoptosis, oncosis, and necrosis. An overview of cell death. Am f Pathol 1995;146:3-15.

9 Germuth FG Jr, Maumenee AE, Senterfit LB, et al. Immunohistologic studies on antigen-antibody reactions in the avascular cornea. F Exp Med 1961;115:919-31.

10 Gobe G, Browning J, Howard T, et al. Apoptosis occurs in endothelial cells during hypertension-induced microvascular rarefaction. $\mathcal{F}$ Struct Biol 1997;118:63-72.

11 Pollman MJ, Naumovski L, Gibbons GH. Endothelial cell apoptosis in capillary network remodeling. $\mathcal{F}$ Cell Physiol 1999;178:359-70.

12 Gavrieli Y, Sherman Y, Ben-Sasson SA. Identification of programmed cell death in situ via specific labeling of nuclear DNA fragmentation. F Cell Biol 1992;119:493501.

13 Horvat R, Palade GE. Thrombomodulin and thrombin localization on the vascular endothelium; their internalization and transcytosis by plasmalemmal vesicles. Eur $\mathcal{F}$ Cell Biol 1993;61:299-313.

14 Esmon CT, Owen WG. Identification of an endothelial cell cofactor for thrombin-catalyzed activation of protein C. Proc Natl Acad Sci 1981;78:2249-52.

15 DeBault LE, Esmon NL, Olson JR, et al. Distribution of the thrombomodulin antigen in the rabbit vasculature. $L a b$ Invest 1986;54:172-87.

16 Streilein JW. Unraveling immune privilege. Science 1995; 270:1158-9

17 Griffith TS, Brunner T, Fletcher SM, et al. Fas ligandinduced apoptosis as a mechanism of immune privilege. Science 1995;270:1189-92.

18 Kilgore KS, Flory CM, Miller BF, et al. The membrane attack complex of complement induces interleukin-8 and monocyte chemoattractant protein-1 secretion from human umbilical vein endothelial cells. Am $\mathcal{f}$ Pathol 1996;149:953-61.

19 Jones J, Morgan BP. Apoptosis is associated with reduced expression of complement regulatory molecules, adhesion molecules and other receptors on polymorphonuclear leucocytes:functional relevance and role in inflammation. Immunology 1995;86:651-60.

20 Liles WC, Kiener PA, Ledbetter JA, et al. Differential expression of Fas (CD95) and Fas ligand on normal human phagocytes: implications for the regulation of apoptosis in neutrophils. F Exp Med 1996;184:429-40.

21 Marshall JC, Watson WG. Programmed cell death (apoptosis) and the resolution of systemic inflammation. CFS 1997;40:169-74.

22 Sang QX. Complex role of matrix metalloproteinases in angiogenesis. Cell Res 1998;8:171-7.

23 ColesHSR, Burne JF, Raff MC. Large-scale normal cell death in the developing rat kidney and its reduction by epidermal growth factor. Development 1993;118:777-84

24 Tsuji S, Kaji K, Nagasawa S. Activation of the alternative pathway of human complement by apoptotic human umbilical vein endothelial cells. $\mathcal{F}$ Biochem 1994;116:794800.

25 Ishizaki M, Zhu G, Haseba T, et al. Expression of collagen I, smooth muscle $\alpha$-actin, and vimentin during the healing of alkali-burned and lacerated corneas. Invest Ophthalmol Vis Sci 1993;34:3320-8.

26 Nagata S. Apoptosis by death factor. Cell 1997;88:355-65.

27 Stuart PM, Griffith TS, Usui N, et al. CD95 ligand (Fas L)-induced apoptosis is necessary for corneal allograft surv)-induced apoptosis is necessary for

28 Nishiuchi R, Yoshino T, Matsuo Y, et al. The Fas antigen is detected on immature B cells and the representative cell lines show Fas-mediated apoptosis. Br f Haematol 1996;92: $302-7$. 